\title{
Reduction of In-Band Interferences' Effect Using Subspace Algorithms in Radio Channel Data
}

\author{
Seda Üstün Ercan ${ }^{* 1}$, Hülya Gökalp ${ }^{2}$
}

Accepted $3^{\text {rd }}$ September 2016

\begin{abstract}
In-band interference increases the noise floor of delay profiles estimated from frequency modulated continuous wave (FMCW) sounder channel data and prevents weak multipath components from being detected. In this study two of the subspace methods named MUltiple SIgnal Classification (MUSIC) and EigenVector (EV) algorithm were used to reduce the effect of interference in delay profiles obtained from an FMCW channel data, and the results are compared with those from conventional FFT method. Results show that MUSIC and EV methods have similar results for time delay estimation, and perform better than the FFT method.
\end{abstract}

Keywords: In-band interference, RF interference, Music, EigenVector, frequency modulated continuous wave.

\section{Introduction}

Using the same band with desired signal, the interfering signals cannot be suppressed at the receiver, hence distorting the receiver output signal. The output of frequency modulated continuous wave (FMCW) channel sounder is sum of sinusoidal signals. But interference results in abrupt fluctuations in the detector output signal and raises the noise floor. The increase in the noise floor may prevent weak multipath components from being detected [1, 2].

In channel sounding, although the signal processing can be performed off-the-line, measurement campaigns are expensive and difficult to repeat. Since every channel data is valuable for a sound channel model, a technique that alleviates the effect of inband-interference in FMCW mobile radio channel data is desirable. Researchers have used various approaches to reduce the effect of in-band interference.

Previous attempts included clipping the level of interference to the level of desired signal or inserting zeros in place of interference corrupted data [3-5], or Prony modelling [6] of data or Minimum (MN) Norm estimate of the channel delay profiles [7].

Subspace methods have been successfully used in estimating frequency components of a signal. Subspace methods are based on Eigen-decomposition of the autocorrelation matrix into signal subspace and noise subspace. Among these methods are Pisarenko harmonic decomposition (PHD), multiple signal classification (MUSIC), Eigenvector (EV) and MN methods. In [8], a novel algorithm based on extended noise subspace MUSIC method was used for Direction Of Arrival (DOA) estimation under the strong interference conditions. In [9] MUSIC and EV are compared for single source; their performances in relation to errors in resolution were found to be similar, and both performed

${ }^{1}$ Department of Electrical and Electronics Engineering, Ondokuz Mayıs University, 55139, Samsun/Turkey

${ }^{2}$ Department of Electrical and Electronics Engineering, Brunel University London, Kingston Lane Uxbridge, London/United Kingdom

* Corresponding Author: Email: sedaustun@hotmail.com

Note: This paper has been presented at the $3^{\text {rd }}$ International Conference on Advanced Technology \& Sciences (ICAT'16) held in Konya (Turkey), September 01-03, 2016.

This journal is @ Advanced Technology \& Science 2013 better than FFT and Pisarenko. For two sources, EV outperformed the other methods in relation to estimation of DOA. In this study MUSIC and EV algorithms are investigated for reducing the effect of interference in FMCW channel data, and the results are compared to those from FFT processing.

\section{Subspace Methods for Frequency Estimation}

Subspace methods are based on eigendecomposition of autocorrelation matrix into signal subspace and noise subspace, and are used for frequency estimation. These methods include PHD, MUSIC, EV MN methods. Pisarenko method uses only one eigenvector. With MUSIC and EV algorithms, the dimension of noise subspace is greater than one, and averaging over noise subspace can be used to improve the performance [10]. These methods are known as high resolution techniques that detect frequency components with a low signal to noise ratios (SNR) [11-12].

\subsection{MUSIC Method}

We assume the received data, $\mathrm{x}(\mathrm{n})$ is a random process consisting of $p$ complex exponentials in white noise with a variance of $\sigma_{w}^{2}$. Such a signal can be written as:

$$
x(n)=\sum_{i=1}^{p} A_{i} e^{-j w_{i} n}+y(n)
$$

where $A_{i}$ is amplitude of the $i$-th complex exponentials and $\mathrm{w}_{i}$ is frequency of the $\mathrm{i}$-th complex exponentials.

$R_{x}$ is the MxM autocorrelation matrix of $x(n)$ with $M>p+1$. If the eigenvalues of $R_{x}$ are arranged in decreasing order ( $\lambda_{1} \geq \lambda_{2} \geq \ldots \geq \lambda_{\mathrm{M}}$ ) and if $\mathrm{v}_{1}, \mathrm{v}_{2}, \ldots, \mathrm{v}_{\mathrm{M}}$ are the corresponding eigenvectors, then these eigenvectors can be divided into two groups: the $p$ signal eigenvectors corresponding to the $p$ largest eigenvalues, and the M-p noise eigenvectors that, ideally, have eigenvalues equal to $\sigma_{w}^{2} \cdot \sigma_{w}^{2}$. This decomposition can be given as follows:

$$
\mathrm{R}_{\mathrm{x}}=\sum_{\mathrm{i}=1}^{\mathrm{M}} \lambda_{\mathrm{i}} v_{\mathrm{i}} v_{\mathrm{i}}^{\mathrm{H}}=\sum_{\mathrm{i}=1}^{\mathrm{p}}\left(\lambda_{\mathrm{i}}^{\mathrm{s}}+\sigma_{\mathrm{w}}^{2}\right) v_{\mathrm{i}} v_{\mathrm{i}}^{\mathrm{H}}+\sum_{\mathrm{i}=\mathrm{p}+1}^{\mathrm{M}} \sigma_{\mathrm{w}}^{2} v_{\mathrm{i}} v_{\mathrm{i}}^{\mathrm{H}}
$$


The white noise variance can be estimated by averaging the $M-p$ smallest eigenvalues as follow:

$$
\sigma_{w}^{2}=\frac{1}{M-p} \sum_{k=p+1}^{M} \lambda_{k}
$$

The MUSIC method frequency estimation function is as follows [7]:

$$
\hat{\mathrm{P}}_{\mathrm{MU}}\left(\mathrm{e}^{\mathrm{jw}}\right)=\frac{1}{\sum_{\mathrm{i}=\mathrm{p}+1}^{\mathrm{M}}\left|\mathbf{e}^{\mathrm{H}} \mathbf{v}_{\mathrm{i}}\right|^{2}}
$$

where $\mathrm{vi}_{\mathrm{i}}$ are the noise eigenvectors and

$$
\mathrm{e}=\left[\begin{array}{lllll}
1 & \mathrm{e}^{\mathrm{jw}} & \mathrm{e}^{\mathrm{j} 2 \mathrm{w}} & \ldots & \mathrm{e}^{\mathrm{j}(\mathrm{M}-1) \mathrm{w}}
\end{array}\right]^{\mathrm{T}} \text { is an arbitrary vector. }
$$

The locations of the $p$ largest peaks of the estimation function give the frequency estimates for the $p$ signal components.

\subsection{EV Method}

The exponential frequencies are estimated in EV algorithm as follows:

$$
\hat{\mathrm{P}}_{\mathrm{EV}}\left(\mathrm{e}^{\mathrm{jw}}\right)=\frac{1}{\sum_{\mathrm{i}=\mathrm{p}+1}^{\mathrm{M}} \frac{1}{\lambda_{\mathrm{i}}}\left|\mathbf{e}^{\mathrm{H}} \mathbf{v}_{\mathrm{i}}\right|^{2}}
$$

The only difference between MUSIC and EV method is the use of inverse eigenvalues. EV method was found to produce fewer spurious peaks [12].

\section{FMCW Channel Data}

FMCW systems are used in radar applications and radio channel propagation measurements [13]. Radio channel propagation data used in the current study was collected in Manchester city center using an FMCW sounder. The center frequency of the transmitter was $1945 \mathrm{MHz}$ with $60 \mathrm{MHz}$ bandwidth. This bandwidth was swept linearly over sweep duration. We used data intervals corresponding to approximately $5 \mathrm{MHz} \mathrm{RF}$ bandwidth for comparing performances of different frequency estimation techniques..

The APDP of the channel data can provide information on the time delay and amplitude of the multipath components. With the FMCW sounding, the detector output is in the form of the sum of sinusoids of different frequencies. Frequencies of these sinusoids correspond to time delays of the multipath components and the amplitudes correspond to the amplitudes of the multipath components. Presence of in-band interference (RF interference) causes abrupt fluctuations in the detector output signal, and increases noise floor which can easily be observed in delay profile estimates of the channel.

Channel data for one whole sweep (i.e. corresponding to $60 \mathrm{MHz}$ $\mathrm{RF}$ band) with interference is shown in Fig. 1.a, interference free section of the sweep in Fig. 1.b, and interference corrupted section of the sweep (both corresponding to $5 \mathrm{MHz}$ RF band) in Fig. 1.c. As it can be seen in Fig.1.c. that the interference caused abrupt fluctuation, and distorted the sum of sinusoidal form. Note that number of samples per sweep depends on the sampling frequency and sweep repetition rate. For this example, sweep repetition rate was $100 \mathrm{~Hz}$, and detector output was sampled at 1 $\mathrm{MHz}$, hence giving 10000 samples per sweep. (a)

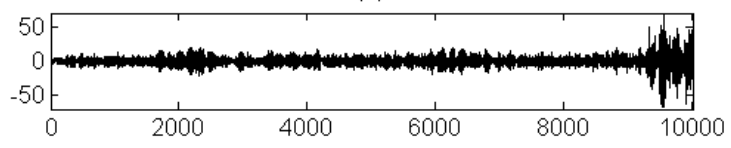

(b)

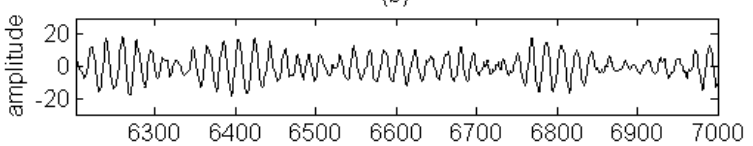

(c)

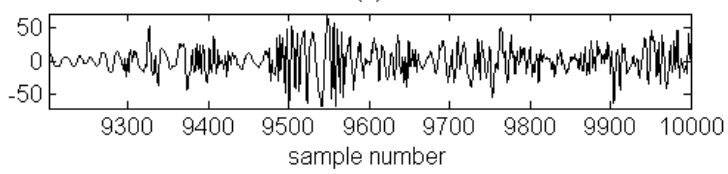

Figure 1. The FMCW receiver output in the time domain

Corresponding average multipath delay profiles for the data given in Fig 1.b and $\mathrm{c}$ are illustrated in Fig.2. These profiles were obtained using FFT method where data from each sweep were Fourier transformed, then magnitude averaged over time (i.e. across sweeps). As it can be seen in Fig.2, in-band interference has increased the noise floor (red) by about $15 \mathrm{~dB}$. This increase in the noise floor obscured weak multipath components with time delays between $13-20 \mu \mathrm{s}$. For the interference-free section (illustrated in black in Figure 2), a few very weak multipath components were detected; one below $-15 \mathrm{~dB}$ at about 14 us time delay and a few below $-20 \mathrm{~dB}$ with various time delays between 17 us and 20 us. These weak multipath components were not identified from the interference-corrupted data (illustrated in red in Fig 2). There are two possible reasons for this: i) these components may be even weaker due to slight difference in propagation mechanisms in the RF band corresponding to this data interval, or ii) the increase in the noise floor obscured the weak multipath components with time delays between $14-20$ us.

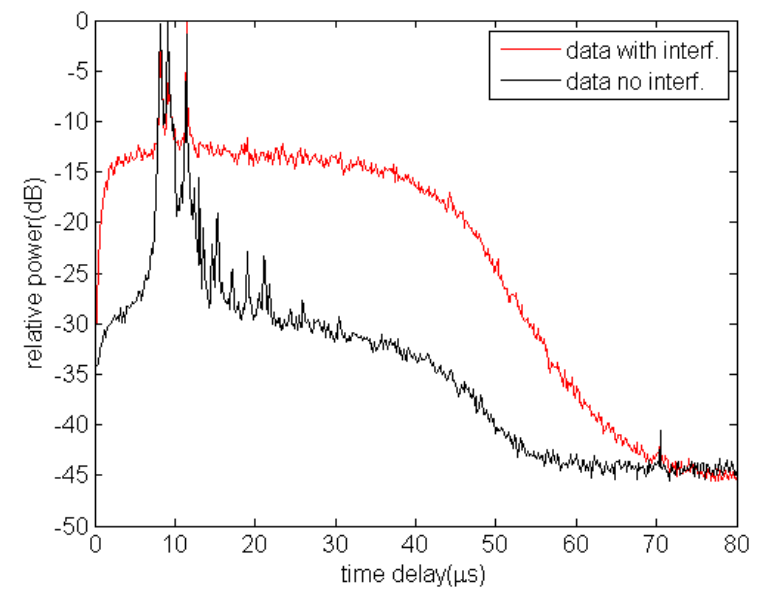

Figure 2. Average PDP estimates for $5 \mathrm{MHz}$ sections with and without interference

\section{Results}

We chose two propagation channel data with in-band interference, and applied FFT method and the two subspace methods (MUSIC and EV) to the data.

\subsection{Channel 1 data}

The detector output data in the time domain and delay profile estimates for this channel data are presented in Fig. 1 and Fig. 2, respectively. Fig. 3 presents the average DP for channel 1 data for FFT (red), MUSIC (black) and EV (blue) methods. As it can be seen, the MUSIC and EV methods considerably reduced the noise 
floor as compared to the FFT method,, the EV method performing slightly better than the MUSIC. However, MUSIC and EV failed to detect the very weak multipath components with times delays of 14 us to 20 us (see results in black in Fig. 2). Looking the at noise floor levels from the MUSIC and EV methods, one would not expect to detect the multipath components below $-20 \mathrm{~dB}$, and it is possible that the component with $-17 \mathrm{~dB}$ relative power and 14 us time delay may be even weaker for this interference-corrupted section [3].

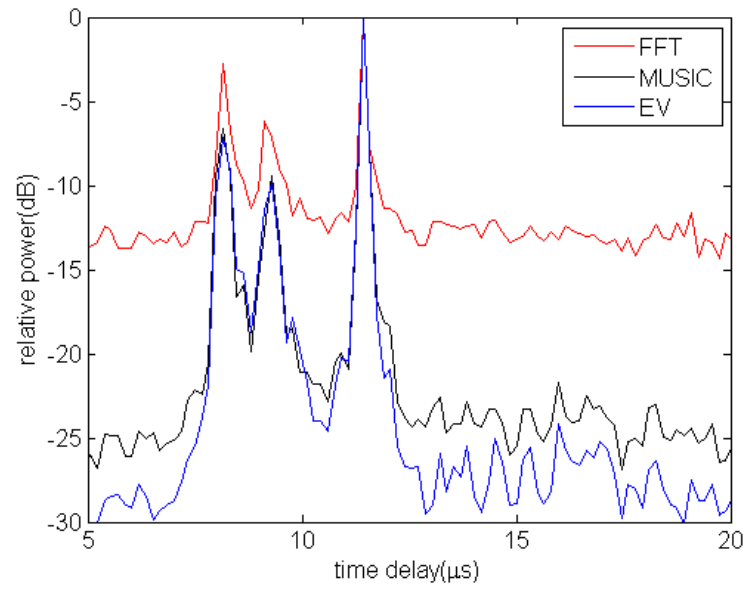

Figure 3. Average DP estimates from the interference corrupted data given in Fig. 1.c: using FFT (red), MUSIC (black) and EV (blue) methods

\subsection{Channel 2 data}

Fig. 4 presents the average DPs for an interference-corrupted section of channel 2 data using the three frequency estimation methods. Multipath components for channel 2 were denser than those for Channel 1, and interference level was not as severe as that of the channel 1. The FFT method (red) resulted in a noise floor level around $-20 \mathrm{~dB}$. The peaks around 6 us and 7.5 us indicates presence of multipath components. However these components are only $3-4 \mathrm{~dB}$ above the noise floor, and would remain under a noise threshold which is usually identified to be at least $5 \mathrm{~dB}$ above the noise floor, and therefore they would not be considered as detected.

On the other hand, the MUSIC and EV methods reduced the noise floor down to a level around $-40 \mathrm{~dB}$, and detected the weak multipath components around 6 us and 7.5 us time delays.

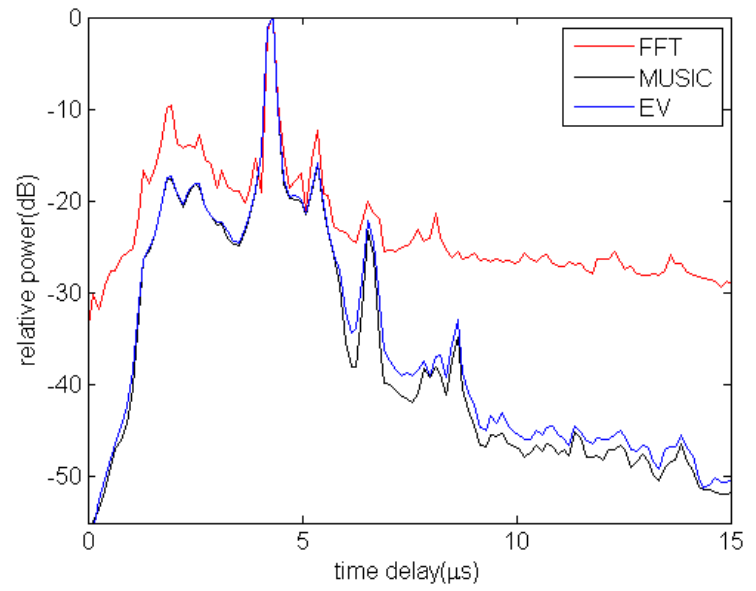

Figure 4. Average DP estimates for channel 2 data using FFT (red), MUSIC (black) and EV (blue) methods

\section{Conclusion}

The results show that the two subspace methods, MUSIC and EV methods, perform better than FFT method with interferencecorrupted data. The two subspace methods enabled detection of weak multipath components which could not be detected by the FFT method.

\section{References}

[1] Goppelt M., Blöcher H.-L., Menzel W. Automotive RadarInvestigation of Mutual Interference Mechanisms, Advances in Radio Science, Volume 8, 2010, 2010, pp.5560 .

[2] Tullsson B. E. Topics in FMWC Radar Disturbance Suppression, IET Conference Publications, 1997.

[3] Salous S., Gokalp H. Medium And Large Scale Characterization Of UMTS Allocated Frequency Division Duplex Channels, IEEE Transactions on Vehicular Technology, 56, 5, 2007, pp. 2831-2843.

[4] Brooker G. M., Mutual Interference of Millimeter Wave Radar Systems, IEEE Transactions on Electromagnetic Compatibility, 49, 1, 2007, pp.170-181.

[5] Schipper T., Harter M., Zwirello L., Mahler T., Zwick T., Systematic approach To Investigate and Counteract Interference-Effects in Automatiive Radars, $9^{\text {th }}$ European Radar Conference (EuRAD), 2012.

[6] Gokalp H., Taflan G.Y., Salous S. In-Band Interference Reduction in FMCW Channel Data Using Prony Modelling, IET Electronic Letters, 45, 2, 2009, pp. 132133.

[7] Gokalp H., Taflan G.Y., Ustun S.,Min-norm Method for Estimating Delay and Doppler Profiles from FMCW Channel Data With In-band Interference, IET Electronic Letters, 40, 11, 2010, pp. 799-800.

[8] Jinyou Qu, Xun Li, Yijun Wen, A New Method for Weak Signals DOA Estimation in the presence of Strong Interferences", 2012 IEEE $11^{\text {th }}$ International Conference on Signal Processing, 2012, pp. 320-323,

[9] Al-Azzo M. F., Al- Sabaawi K. High Resolution Techniques for Direction of Arrival Estimation of Ultrasonic Waves, American Journal of Signal Processing, 2014, pp. 49-59.

[10] Schmidt R. O., "A Signal Subspace Approach to Multiple Emitter Location and Spectral Estimation", PhD thesis, Stanford University, Stanford, CA, 1981.

[11] Youn Y. W., Yi S. H., Hwang D. H., Sun J. H., Kang D. S., Kim Y. H., "MUSIC-based diagnosis algorithm for identifying broken rotor bar faults in induction motors using flux signal", Journal of Electrical Engineering \& Technology, 8.2, 2013, pp. 288-294.

[12] Chakkor S., Baghouri M., Hajraoui A., "Performance Analysis of Faults Detection in Wind Turbine Generator Based on High-Resolution Frequency estimation Methods", International Journal of Advanced Computer Science and Applications, 5, 4, 2014, pp. 139-148.

[13] Gokalp H., Characterization of UMTS FDD Channels, Science and Technology for the degree of Doctor of Philosophy, Department of Electrical Engineering and Electronics, Manchester, United Kingdom, 2001. 